\title{
Fibers of the $L^{\infty}$ algebra and disintegration of measures
}

\author{
MareK Kosiek And Krzysztof Rudol
}

\begin{abstract}
It is shown that Gelfand transforms of elements $f \in L^{\infty}(\mu)$ are almost constant at almost every fiber $\Pi^{-1}(\{x\})$ of the spectrum of $L^{\infty}(\mu)$ in the following sense: for each $f \in L^{\infty}(\mu)$ there is an open dense subset $U=U(f)$ of this spectrum having full measure and such that the Gelfand transform of $f$ is constant on the intersection $\Pi^{-1}(\{x\}) \cap U$. As an application a new approach to disintegration of measures is presented, allowing one to drop the usually taken separability assumption.
\end{abstract}

Mathematics Subject Classification (2010). Primary 46J10, 28A50; Secondary 46E30, 28A20.

Keywords. Function algebra, Measure, $L^{\infty}$ algebra, Fiber, Disintegration.

1. Introduction. Let $\mu$ be a Borel measure on a compact topological space $X$. The Gelfand spectrum of the algebra $L^{\infty}(\mu)$ despite of being compact, is in general quite large. Among many interesting properties - it has a natural fiber-wise structure determined by the constant values of Gelfand transforms $\widehat{[f]}$ of elements $[f] \in L^{\infty}(\mu)$ corresponding to continuous functions $f$ on $X$. Our main result (due to the first-named author) says that on some "large" sets, all elements $h \in L^{\infty}(\mu)$ behave in much the same manner as in the continuous case. The proof bases on topological and measure properties of the spectrum of $L^{\infty}(\mu)$, (see [2], [3, I.9]) and is related to abstract approach to A-measures problem and corona problem.

As an application, we prove in Section 3 a disintegration theorem for regular Borel complex measures on compact spaces. By the results of Section 2 it is possible to drop the usually taken separability assumption and get a relatively simple proof. In the final section - using the disintegration theorem we look at the main result from a slightly different perspective.

Marek Kosiek was supported by Ministry of Science and Higher Education Grant NN201 546438 . 
2. Fibers of the $\boldsymbol{L}^{\infty}$ algebra. In this section we consider a probabilistic Borel measure $\mu$ on a compact topological space $X$, assuming that

(*) $\mu$ is regular and $X$ is equal to the closed support of $\mu$.

The set $L^{\infty}(\mu)$ of equivalence classes $[f]$ of essentially bounded $\mu$-measurable functions $f$ on $X$ is a commutative $\mathrm{C}^{*}$-algebra under standard operations.

Let $Y$ be the spectrum of $L^{\infty}(\mu)$. By Gelfand-Naimark theorem, $L^{\infty}(\mu)$ is isometrically isomorphic (by the Gelfand transform $[f] \rightarrow \widehat{[f]}$ ) to the Banach algebra $C(Y)$ of all continuous, complex-valued functions on $Y$.

In our setting there is a natural "projection map" $\Pi: Y \rightarrow X$ constructed as follows: The points $y \in Y$ correspond to the functionals $\Pi_{y}$ defined on $C(X)$ by

$$
\Pi_{y}(f):=\widehat{[f]}(y) \text { for } f \in C(X) .
$$

As a composition of the embedding of $C(X)$ in $L^{\infty}(\mu)$ and of $y: L^{\infty}(\mu) \ni h \rightarrow$ $\hat{h}(y)=y(h) \in \mathbb{C}$, the functional $\Pi_{y}$ is linear-multiplicative on $C(X)$. Hence it can be identified with some point $\Pi(y)$ in $X$, so that for any $f \in C(X)$ we have $f(\Pi(y))=\Pi_{y}(f)$, i.e. $f \circ \Pi=\widehat{[f]}$. Hence $f \circ \Pi$ is a continuous function on $Y$ for each $f \in C(X)$. To clarify the setting, let us collect some simple observations.

Proposition 2.1. $\quad$ 1. The projection $\Pi: Y \rightarrow X$ is continuous and surjective.

2. Up to the isometry $f \rightarrow[f], C(X)$ can be considered as a closed subalgebra of $L^{\infty}(\mu)$.

3. Each element $x \in X$ as a linear-multiplicative functional on $C(X)$ has a linear-multiplicative extension $y:[f] \rightarrow \widehat{[f]}(y)$ to the whole $L^{\infty}(\mu)$, and for any such an extension $\Pi(y)=x$, and in this sense one can view $\Pi$ as a projection.

Proof. Since the Gelfand topology on $X$ is induced by the weak-star topology with $X$ treated as a subset of the dual of $C(X)$, the continuity of $\Pi$ follows from the continuity of $f \circ \Pi$ for all $f \in C(X)$. The isometry of $C(X) \ni f \rightarrow$ $[f] \in L^{\infty}(\mu)$ follows from $(*)$, hence all the mappings in the sequence

$$
C(X) \ni f \rightarrow[f] \rightarrow \widehat{[f]} \in C(Y)
$$

are isometric (the second one is the Gelfand transform), and by (2.1) we have for $f \in C(X)$

$$
\sup _{x \in X}|f(x)|=\|f\|=\|\widehat{[f]}\|=\sup _{x \in \Pi(Y)}|f(x)| .
$$

As a continuous image of the compact space $Y$, the set $\Pi(Y)$ is compact, and hence closed in $X$ which by (2.3) implies that $\Pi(Y)$ contains the Shilov boundary of $C(X)$. Consequently $\Pi(Y)$ must be equal to $X$. Surjectivity comes also from the last claim, easy to establish. Note that all the extensions $y$ of the given $x$ form the set equal to the fiber $\Pi^{-1}(\{x\})$. 
From now on we will not distinguish in writing between $[\mu]$-essentially bounded Borel functions on $X$ and their equivalence classes in $L^{\infty}(\mu)$. We have seen that $\widehat{f}$ is constant on each fiber $\Pi^{-1}(\{x\})$ for any $f \in C(X)$.

Since we identify $L^{\infty}(\mu)$ with $C(Y)$, the Riesz Representation Theorem gives a regular positive Borel measure $\tilde{\mu}$ on $Y$ "representing $\mu$ " in the sense that $\|\tilde{\mu}\|=\|\mu\|$ and

$$
\int f d \mu=\int \hat{f} d \tilde{\mu} \text { for } f \in L^{\infty}(\mu) .
$$

For any Borel $E \subset X$, the Gelfand transform $\widehat{\chi_{E}}$ of its characteristic function $\chi_{E}$, as an idempotent in $C(Y)$, is of the form $\chi_{U_{E}}$, thus assigning a closed-open set $U_{E}$ in $Y$ to any measurable $E \subset X$. Applying (2.4) to $\chi_{E}$ we get for any Borel subset $E$ of $\mathrm{X}$ the equality

$$
\mu(E)=\tilde{\mu}\left(U_{E}\right) .
$$

Moreover (Lemma 9.1 and Corollary 9.2 of [3]) we have

Lemma 2.2. The family $\left\{U_{E}: E \subset Y, E\right.$ measurable $\}$ forms a basis for the topology of $Y$. If $U$ is an open non-empty subset of $Y$, then $\tilde{\mu}(U)>0$.

Lemma 2.3. If $E, F$ are Borel subsets of $X$ and $E \subset F$, then $\widehat{\chi_{E}} \leq \widehat{\chi_{F}}$ and $U_{E} \subset U_{F}$.

Proof. If $E \subset F$ then $\chi_{E}=\chi_{E} \cdot \chi_{F}$. Hence $\widehat{\chi_{E}}=\widehat{\chi_{E}} \cdot \widehat{\chi_{F}}$ which means that $\widehat{\chi_{E}} \leq \widehat{\chi_{F}}$. Since $\chi_{U_{E}}=\widehat{\chi_{E}}$ and $\chi_{U_{F}}=\widehat{\chi_{F}}$, we have $U_{E} \subset U_{F}$.

Lemma 2.4. If $E \subset X$ is open then $\Pi^{-1}(E) \subset U_{E}$ and $\chi_{\Pi^{-1}(E)} \leq \widehat{\chi_{E}}$. If $E \subset X$ is closed, then $\Pi^{-1}(E) \supset U_{E}$ and $\chi_{\Pi^{-1}(E)} \geq \widehat{\chi_{E}}$.

Proof. Let $E$ be open in $X$ and $x \in E$. Then there is a continuous function $f: X \rightarrow[0,1]$ such that $f(x)=1$ and $f \leq \chi_{E}$. Hence $\hat{f}$ is equal 1 on $\Pi^{-1}(\{x\})$ and $f=f \cdot \chi_{E}$, which implies $\hat{f}=\hat{f} \cdot \widehat{\chi_{E}}=\hat{f} \cdot \chi_{U_{E}}$. Consequently $\widehat{\chi_{U_{E}}}$ is equal 1 on $\Pi^{-1}(\{x\})$ which means that $\Pi^{-1}(\{x\}) \subset U_{E}$. Since $x$ was an arbitrary point of $E$, we have $\Pi^{-1}(E) \subset U_{E}$. Then also $\chi_{\Pi^{-1}(E)} \leq \chi_{U_{E}}=\widehat{\chi_{E}}$.

If $E$ is closed then $X \backslash E$ is open and $\chi_{E} \cdot \chi_{X \backslash E}=0, \chi_{E}+\chi_{X \backslash E}=1$. Consequently $\chi_{U_{E}} \chi_{U_{X \backslash E}}=\widehat{\chi_{E}} \cdot \widehat{\chi_{X \backslash E}}=0$ and $\chi_{U_{E}}+\chi_{U_{X \backslash E}}=\widehat{\chi_{E}}+\widehat{\chi_{X \backslash E}}=1$. It means that $U_{E} \cap U_{X \backslash E}=\emptyset$ and $U_{E} \cup U_{X \backslash E}=Y$ which implies the desired statement for closed sets.

Remark 2.5. Till now the regularity of $\mu$ has not been used.

Lemma 2.6. If $E$ is a Borel subset of $X$ then

$$
\mu(E)=\tilde{\mu}\left(\Pi^{-1}(E)\right)=\tilde{\mu}\left(U_{E}\right) .
$$

If $E \subset X$ is open then $\overline{\Pi^{-1}(E)}=U_{E}$. If $E \subset X$ is closed then $\operatorname{int}\left(\Pi^{-1}(E)\right)=$ $U_{E}$.

Proof. By the regularity of $\mu$, for any $\varepsilon>0$ we can find a compact set $K \subset X$ and an open set $V \subset X$ such that $K \subset E \subset V$ and $\mu(V \backslash K)<\varepsilon$. Also, there exists $f \in C(X)$ such that $\chi_{K} \leq f \leq \chi_{V}$. By the continuity of $f$ we have $\chi_{\Pi^{-1}(K)} \leq \hat{f} \leq \chi_{\Pi^{-1}(V)}$. (Proposition 2.1 and the consideration following it). 
Hence $\left|\mu(E)-\int f d \mu\right|<\varepsilon$ and $\left|\tilde{\mu}\left(\Pi^{-1}(E)\right)-\int \hat{f} d \tilde{\mu}\right|<\varepsilon$ which by (2.4) and by the arbitrariness of the choice of $\varepsilon$ - gives $\mu(E)=\tilde{\mu}\left(\Pi^{-1}(E)\right)$. The second equality in (2.6) we get by (2.5).

If $E$ is closed then $U_{E} \subset \Pi^{-1}(E)$ by Lemma 2.4. So $U_{E} \subset \operatorname{int}\left(\Pi^{-1}(E)\right)$ and $\operatorname{int}\left(\Pi^{-1}(E)\right) \backslash U_{E}$ is open since $U_{E}$ is closed-open. Consequently, we have $\operatorname{int}\left(\Pi^{-1}(E)\right)=U_{E}$ by Lemma 2.2 .

The assertion for open sets follows from the equalities $\operatorname{int}\left(\Pi^{-1}(E)\right)=$ $Y \backslash \overline{\Pi^{-1}(X \backslash E)}$ and $U_{E}=Y \backslash U_{X \backslash E}$.

Theorem 2.7. If $\mu$ is a probabilistic measure satisfying (*), $Y$ is the spectrum of $L^{\infty}(\mu)$, and $h \in L^{\infty}(\mu)$, then there exists an open dense subset $U$ of $Y$ with $\tilde{\mu}(U)=\tilde{\mu}(Y)$ such that $\hat{h}$ is constant on $\Pi^{-1}(\{x\}) \cap U$ for all $x \in X$.

Proof. Let $h \in L^{\infty}(\mu)$, and let $\varepsilon>0$. By Lusin Theorem there is $g \in C(X)$ with $\|g\| \leq\|h\|$ and a closed set $Z \subset X$ such that $\mu(X \backslash Z)<\varepsilon$ while $Z \subset$ $\{g=h\}$. By Lemma 2.6 we have

$$
U_{Z}=\operatorname{int}\left(\Pi^{-1}(Z)\right), \quad \tilde{\mu}\left(U_{Z}\right)=\mu(Z)>1-\varepsilon .
$$

Since $Z \subset\{g=h\}$ then $\chi_{Z} \cdot(g-h)=0$. Consequently $\chi_{U_{Z}} \cdot(\hat{g}-\hat{h})=$ $\widehat{\chi Z} \cdot(\hat{g}-\hat{h})=0$ which implies

$$
\{\hat{g} \neq \hat{h}\} \cap U_{Z}=\emptyset .
$$

Put $Z_{1}:=Z$ and $\varepsilon=1 / 2$. Repeating the previous construction we find a sequence $\left\{g_{n}\right\} \subset C(X)$ and a sequence $\left\{Z_{n}\right\}$ of closed subsets of $X$ such that $Z_{n} \subset\left\{g_{n}=h\right\}$ and $\mu\left(X \backslash Z_{n}\right)<1 / 2^{n}$. Then

$$
\tilde{\mu}\left(U_{Z_{n}}\right)=\mu\left(Z_{n}\right)>1-1 / 2^{n}, \quad\left\{\hat{g}_{n} \neq \hat{h}\right\} \cap U_{Z_{n}}=\emptyset .
$$

The last equality implies that $\hat{h}$ is constant on each $\Pi^{-1}(\{x\}) \cap U_{Z_{n}}$ for all $x \in X$ and $n \in \mathbb{N}$. We define a sequence of open sets as follows:

$$
U_{1}:=U_{Z_{1}}, \quad U_{n}:=U_{Z_{n}} \backslash \Pi^{-1}\left(Z_{1} \cup \cdots \cup Z_{n-1}\right) .
$$

By the above definition and Lemma 2.4, for $k \in \mathbb{N}$ we have $\Pi^{-1}\left(Z_{k}\right) \supset U_{Z_{k}} \supset$ $U_{k}$, hence $Z_{k} \supset \Pi\left(U_{Z_{k}}\right) \supset \Pi\left(U_{k}\right)$, and consequently

$$
\Pi\left(U_{n}\right) \cap \Pi\left(U_{m}\right)=\emptyset \quad \text { for } n \neq m
$$

since $\Pi\left(U_{n}\right) \cap Z_{k}=\emptyset$ for $k<n$. By Lemma 2.6 we have $\tilde{\mu}\left(\Pi^{-1}\left(Z_{n}\right) \backslash U_{Z_{n}}\right)=0$ and hence

$$
\begin{aligned}
\tilde{\mu}\left(U_{n}\right) & =\tilde{\mu}\left(U_{Z_{n}} \backslash \Pi^{-1}\left(Z_{1} \cup \cdots \cup Z_{n-1}\right)\right)=\tilde{\mu}\left(\Pi^{-1}\left(Z_{n}\right) \backslash \Pi^{-1}\left(Z_{1} \cup \cdots \cup Z_{n-1}\right)\right) \\
& =\tilde{\mu}\left(\Pi^{-1}\left(Z_{n} \backslash\left(Z_{1} \cup \cdots \cup Z_{n-1}\right)\right)=\mu\left(Z_{n} \backslash\left(Z_{1} \cup \cdots \cup Z_{n-1}\right)\right) .\right.
\end{aligned}
$$

Put now $Z_{1}^{\prime}:=Z_{1}$ and $Z_{n}^{\prime}:=Z_{n} \backslash\left(Z_{1} \cup \cdots \cup Z_{n-1}\right)$ for $n>1$. All the sets $\left\{Z_{n}^{\prime}\right\}$ are pairwise disjoint and a direct calculation gives the equality $Z_{n}^{\prime} \cup Z_{n-1}^{\prime} \supset$ $Z_{n} \backslash\left(Z_{1} \cup \cdots \cup Z_{n-2}\right)$ which by induction leads to the assertion $Z_{1}^{\prime} \cup \cdots \cup Z_{n}^{\prime} \supset Z_{n}$. Hence, by (2.8) and pairwise disjointness of $\left\{U_{n}\right\}$ and $\left\{Z_{n}^{\prime}\right\}$, we get

$$
\begin{aligned}
\tilde{\mu}\left(U_{1} \cup \cdots \cup U_{n}\right) & =\tilde{\mu}\left(U_{1}\right)+\cdots+\tilde{\mu}\left(U_{n}\right)=\mu\left(Z_{1}^{\prime}\right)+\cdots+\mu\left(Z_{n}^{\prime}\right) \\
& =\mu\left(Z_{1}^{\prime} \cup \cdots \cup Z_{n}^{\prime}\right) \geq \mu\left(Z_{n}\right)>1-1 / 2^{n} .
\end{aligned}
$$


Put $U:=\bigcup_{n=1}^{\infty} U_{n}$. Hence $U$ is open, $\tilde{\mu}(U)=1=\tilde{\mu}(Y)$, and consequently, by Lemma 2.2, $U$ is dense in $Y$. The function $\hat{h}$ is constant on each $\Pi^{-1}(\{x\}) \cap U_{n}$ for all $x \in X$ and $n \in \mathbb{N}$ and sets $\Pi\left(U_{n}\right), n \in \mathbb{N}$ are pairwise disjoint by (2.7). It means that each fiber $\Pi^{-1}(\{x\})$ intersects at most one of the sets $U_{n}$. Hence $\hat{h}$ is constant on each $\Pi^{-1}(\{x\}) \cap U$ for all $x \in X$.

Remark 2.8. If the closed support of $\mu$ is not equal to $X$, then $L^{\infty}(\mu)$ is isometrically isomorphic to the algebra $\left\{f_{\mid \operatorname{supp}(\mu)}: f \in L^{\infty}(\mu)\right\}$. In such a case $\Pi^{-1}(\{x\})=\emptyset$ for all $x$ outside of the closed support of $\mu$. Assuming that each function is constant on empty set we conclude that the result of Theorem holds true also when the closed support of $\mu$ is a proper subset of $X$.

3. Disintegration of measures. In this section $X, Y, Z$ will be compact spaces, and the word "measurable" will concern their Borel sigma-fields $\mathcal{B}_{X}, \mathcal{B}_{Y}, \mathcal{B}_{Z}$. Given a complex Borel measure $\nu$ on $X$ and a measurable mapping $P: X \rightarrow Z$ we denote by $P(\nu)$ the pushforward measure defined on $Z$ by

$$
P(\nu)(E):=\nu\left(P^{-1}(E)\right), \quad E \in \mathcal{B}_{Z},
$$

so that

$$
\int_{Z} h d P(\nu)=\int_{X}(h \circ P) d \nu, \quad h \in C(Z) .
$$

Denote by $\mu$ the measure $P(|\nu|)$ and assume (without loss of generality) that its total variation norm satisfies $\|\mu\|=1$.

Let us recall that for a family of measures $\nu_{z}, z \in Z$ the vector-valued integral $\int_{Z} \nu_{z} d \mu$ is the measure $\nu$ such that for any continuous function $h$ on $X$ we have

$$
\int h d \nu=\int_{Z}\left(\int h(x) d \nu_{z}(x)\right) d \mu(z) .
$$

The disintegration of a Borel probability measure $\nu$ on a compact space $X$ with respect to a mapping $P: X \rightarrow Z$ is a measurable family of probability measures $\nu_{z}$ satisfying (3.1) and carried by the fibers $P^{-1}(\{z\})$. The existence of disintegration under certain assumptions including the separability of $X$ is shown in [1]. Our approach is to build the measures $\nu_{z}$ using certain properties of the Gelfand spectrum $Y$ of the Banach algebra $L^{\infty}(\mu)$. If $\nu$ is a complex Borel measure, one can still obtain (3.1), allowing the $\nu_{z}$ to be complex measures. Our proof implies that $\nu_{z}$ are supported on $P^{-1}(\{z\})$.

Let us begin by fixing some notation. Given a continuous function $f \in$ $C(X)$, denote by

$$
g_{f}=g_{f}^{\nu}:=\frac{d(P(f \nu))}{d(P(|\nu|))}
$$

the Radon-Nikodym derivative of the pushforward measures for " $\nu$ times density $f$ " with respect to that of the variation measure $|\nu|$. The shorthand notation $g_{f}$ will be used rather than $g_{f}^{\nu}$ if the measure $\nu$ is clear from the context. 
Bearing in mind their absolute continuity, we obtain for any $\psi \in L^{1}(\mu)$ (recall that $\mu=P(|\nu|)$ ) the equalities

$$
\int_{Z} \psi(z) g_{f}(z) d \mu(z)=\int_{Z} \psi(z) d(P(f \nu))(z)=\int_{X} \psi(P(x)) f(x) d \nu(x)
$$

Clearly, we have $g_{f} \in L^{1}(\mu)$.

Lemma 3.1. For any $f \in C(X)$ we have $g_{f} \in L^{\infty}(\mu)$ and $\left\|g_{f}\right\|_{\infty} \leq\|f\|$.

Proof. Let $h \in L^{1}(\mu)$. Then, as in (3.3), using the equality $\mu=P(|\nu|)$ we get

$$
\begin{aligned}
\left|\int h g_{f} d(\mu)\right| & =\left|\int h d(P(f \nu))\right|=\left|\int(h \circ P) f d \nu\right| \\
& \leq\|f\| \int|h \circ P| d|\nu|=\|f\| \int|h| d(P(|\nu|))=\|f\|\|h\|_{1} .
\end{aligned}
$$

So $g_{f}$ as a functional on $L^{1}(\mu)$ has its norm estimated by $\|f\|$ (the sup-norm over $X$ ) and the result follows.

Assume, for convenience reasons that $g_{f}$ is real. (The general case will easily follow by splitting into the real and imaginary parts and multiplying by a constant.) As in the previous section, let $Y$ be the spectrum of the Banach algebra $L^{\infty}(\mu)$. It is a totally disconnected compact space with its Gelfand topology.

Let $\Pi: Y \rightarrow Z$ be the canonical projection (cf. Section 2 and [3]) that assigns to a multiplicative linear functional $y \in Y$ a unique point $\Pi_{y} \in Z$ so that for any $f \in C(Z)$ one has $f\left(\Pi_{y}\right)=y([f])$. The measure $\mu$ lifts to a Borel measure $\tilde{\mu}$ on $Y$ so that $\Pi(\tilde{\mu})=\mu$. As follows from Sections 2 and [3], such a Borel measure on $Y$ is actually unique. Theorem 2.7 provides for arbitrarily chosen $h \in L^{\infty}(\mu)$ (here $h=g_{f}$ ) a dense open set $U=U_{h}$ in $Y$, having full measure $\tilde{\mu}$ and such that $\widehat{h}$ is constant on each set $\Pi^{-1}(\{z\}) \cap U_{h}$ for $z \in Z$.

For $z \in Z$ denote

$$
\mathcal{U}_{z}:=\left\{\Pi^{-1}(\Pi(V)): V \subset Y, V \text { closed-open, } z \in \Pi(V)\right\} .
$$

For any $z \in Z$ we define a linear functional $\Phi_{z}: C(X) \rightarrow \mathbb{R}$ putting

$$
\Phi_{z}(f):=\operatorname{Lim}_{E \in \mathcal{U}_{z}} \frac{1}{\tilde{\mu}(E)} \int_{E} \widehat{g_{f}} d \tilde{\mu} .
$$

Here Lim denotes a Banach limit. We require it only to be linear and located between the lower- and upper limits with respect to the directed family $\mathcal{U}_{z}$. By Lemma 3.1, $\Phi_{z}$ is bounded, of norm less than or equal 1. Hence for each $z \in Z$ there exists a regular complex Borel measure $\nu_{z}$ on $X$ such that

$$
\Phi_{z}(f)=\int f d \nu_{z} \quad \text { for } \quad f \in C(X), \quad\left\|\nu_{z}\right\| \leq 1 \quad \text { for } \quad z \in Z
$$

Lemma 3.2. For $a \in \Pi^{-1}(\{z\}) \cap U_{g_{f}}$ we have $\Phi_{z}(f)=\widehat{g_{f}}(a)$. 
Proof. Let $a \in \Pi^{-1}(\{z\}) \cap U$, where $U=U_{g_{f}}$. For an arbitrary $\varepsilon>0$ take a closed-open neighbourhood $V_{\epsilon}$ of $a$ such that $\left|\widehat{g_{f}}(y)-\widehat{g_{f}}(a)\right|<\varepsilon$ for $y \in V_{\varepsilon}$ and put $E_{\varepsilon}:=\Pi^{-1}\left(\Pi\left(V_{\varepsilon}\right)\right)$. This is possible since clopen sets form a base of topology for $Y$ (cf. [3]). Since $\widehat{g_{f}}$ is constant on each fiber intersected with $U$ we also have $\left|\widehat{g_{f}}(y)-\widehat{g_{f}}(a)\right|<\varepsilon$ for $y \in E_{\varepsilon} \cap U$. But as we have $\tilde{\mu}(Y \backslash U)=0$, the integral means over the sets $E$ and $E \cap U$ are equal (for $d \tilde{\mu}$ ). The above estimate by $\varepsilon$ for $\widehat{g_{f}}-\widehat{g_{f}}(a)$ yields the same bound $\varepsilon$ for the differences between the integral means over any $E \in \mathcal{U}_{z}$ such that $E \subset E_{\varepsilon}$. Passing to the Banach limits, we get

$$
\left|\Phi_{z}(f)-\widehat{g_{f}}(a)\right| \leq \varepsilon .
$$

Since $\varepsilon$ was arbitrary we get $\Phi_{z}(f)=\widehat{g_{f}}(a)$.

If one considers probability measures $\nu$, for constant function $f_{0}=1$ one has $g_{f_{0}}=1$ and $\Phi_{z}\left(f_{0}\right)=1$, hence our measures $\nu_{z}$ obtained in (3.5) are probabilistic. For complex measures $\nu$ the integral representation (3.1) still has its meaning and we may call it the disintegration of $\nu$ in this general case.

We are now in position to state our main result

Theorem 3.3. The family of measures $\nu_{z}, z \in Z$ satisfies (3.1). Moreover, it forms a disintegration of the measure $\nu$ with respect to $P$, and for any $z \in Z$ the measure $\nu_{z}$ is concentrated on $P^{-1}(\{z\})$.

Proof. Let $E$ be a closed subset of $\mathbb{C}$ and let $\tilde{E}$ be its preimage under the mapping $\left\{z \rightarrow \Phi_{z}(f)\right\}$ i.e.

$$
\tilde{E}=\left\{z \in Z: \Phi_{z}(f) \in E\right\} .
$$

Denote $F:=\Pi\left({\widehat{g_{f}}}^{-1}(E)\right)$. Then

$$
F=\left\{\Pi(a): \widehat{g_{f}}(a) \in E\right\} .
$$

Hence, by Lemma 3.2, $F \cap \Pi\left(U_{g_{f}}\right)=\tilde{E} \cap \Pi\left(U_{g_{f}}\right)$. Since ${\widehat{g_{f}}}^{-1}(E)$ is closed by the continuity of $\widehat{g_{f}}$ and consequently compact, $F$ is also compact. So $\tilde{E}$ differs from $F$ by a set of $[P(|\nu|)]$ measure 0 and consequently is measurable.

Taking $\psi=1$ in (3.3), using (3.5) we get for $f \in C(X), U=U_{g_{f}}$ the equalities

$$
\begin{aligned}
\int_{X} f d \nu & =\int_{Z} g_{f} d \mu=\int_{Y} \widehat{g_{f}} d \tilde{\mu}=\int_{Y \cap U} \widehat{g_{f}}(a) d \tilde{\mu}(a) \\
& =\int_{Y \cap U} \Phi_{\Pi(a)}(f) d \tilde{\mu}(a)=\int_{Y} \Phi_{\Pi(a)}(f) d \tilde{\mu}(a) \\
& =\int_{Z} \Phi_{z}(f) d \mu(z)=\int_{Z}\left(\int f d \nu_{z}\right) d \mu(z)=\int_{Z}\left(\int f d \nu_{z}\right) d(P(|\nu|))(z) .
\end{aligned}
$$

It remains to show that $\nu_{z}$ is carried by $X_{z}:=P^{-1}(\{z\})$ for any $z \in Z$.

Let us begin with the case of non-negative $\nu$. Then for $h \in C(Z)$, denoting $f:=h \circ P$ we get $g_{f}=h$, since $P(f \cdot \nu)=h \cdot P(\nu)$. Now by Lemma 3.2, $\Phi_{z}(f)=$ $h(z)$, since $h$ is continuous. But this gives us the equality $\int f d \nu_{z}=h(z)$ for 
all continuous $h: Z \rightarrow \mathbb{C}$, meaning that $P\left(\nu_{z}\right)$ is the point mass 1 measure $\delta_{z}$ at $z$, proving that $\nu_{z}$ is carried by $P^{-1}(\{z\})$.

In the general case, denote by $\nu_{z}^{\prime}$ the measures (carried by $P^{-1}(\{z\})$ ) obtained by disintegrating $|\nu|$ with respect to $P$. For any nonnegative continuous function $f$ on $X$ we have $|f \nu|=f|\nu|$ and since

$$
|P(f \nu)| \leq P(|f \nu|)=P(f|\nu|),
$$

we have the corresponding inequality for the numerators in (3.2) for $\left|g_{f}^{\nu}\right|$ and $g_{f}^{|\nu|}$-respectively, showing that

$$
\left|g_{f}^{\nu}\right| \leq g_{f}^{|\nu|}
$$

Applying these inequalities for all such non-negative $f \in C(X)$, in (3.4) and (3.5), we get

$$
\left|\int f d \nu_{z}\right| \leq \int f d \nu_{z}^{\prime}
$$

which shows that

$$
\left|\nu_{z}\right| \leq \nu_{z}^{\prime}
$$

and consequently, the $\nu_{z}$ are also carried by $P^{-1}(\{z\})$.

4. Fibers and disintegration. Let now, as in Section 2, $X$ be a compact space $\mu$ be a measure on $X$ satisfying $(*)$, and $Y$ be the spectrum of the algebra $L^{\infty}(\mu)$. By Theorem 3.3, there is a family $\left\{\nu_{x}\right\}_{x \in X}$ of Borel regular measures on $Y$ such that

$$
\int \hat{f} d \tilde{\mu}=\int_{X}\left(\int \hat{f}(y) d \nu_{x}(y)\right) d \mu(x)
$$

for $f \in L^{\infty}(\mu)$ (i.e $\hat{f} \in C(Y)$ ), and each $\nu_{x}$ is carried by $\Pi^{-1}(\{x\})$ for any $x \in X$. Since $\mu$ is probabilistic, the formulas (3.4) and (3.5) used for the function identically equal to 1 , give $\nu_{x}(X)=1$ and $\left\|\nu_{x}\right\| \leq 1$, which implies that each $\nu_{x}$ is non-negative. Recall from Section 2 that to any Borel set $E \subset X$ we can uniquely assign by the Gelfand transform of its characteristic function a closed-open set $U_{E} \subset Y$.

Proposition 4.1. For any $f \in L^{\infty}(\mu)$ there is sequence of Borel subsets $\left\{E_{n}\right\}_{n=1}^{\infty} \subset X$ such that $U_{f}:=\bigcup_{n=1}^{\infty} U_{E_{n}}$ is an open dense subset of $Y$ with $\tilde{\mu}\left(U_{f}\right)=1$ and $\hat{f}$ is constant on $\Pi^{-1}(\{x\}) \cap U_{f}$ for all $x \in X$.

Proof. Take an arbitrary $f \in L^{\infty}(\mu)$. By Theorem 2.7 there is an open dense subset $U$ of $Y$ with $\tilde{\mu}(U)=1$ and such that $\hat{f}$ is constant on $\Pi^{-1}(\{x\}) \cap U$ for all $x \in X$. By the regularity of $\tilde{\mu}$ we can find a compact set $K \subset U$ such that $\tilde{\mu}(U \backslash K)<1 / 2$. Since $K$ is compact, we can find a finite collection $\left\{F_{i}\right\}_{i=1}^{k}$ of Borel subsets of $X$ such that $K \subset \bigcup_{i=1}^{k} U_{F_{i}} \subset U$. Put $E_{1}:=\bigcup_{i=1}^{k} F_{i}$. Then $U_{E_{1}}=\bigcup_{i=1}^{k} U_{F_{i}} \subset U$ and $\tilde{\mu}\left(U \backslash U_{E_{1}}\right)<1 / 2$. By induction we find a sequence of Borel sets $E_{n} \subset X$ such that $U_{E_{n}} \subset U$ and

$$
\tilde{\mu}\left(U \backslash U_{E_{n}}\right)<1 / 2^{n} .
$$


Replacing each $U_{E_{n}}$ by $\bigcup_{i=1}^{n} U_{E_{i}}$ we get an increasing sequence of closed-open subsets of $U$ satisfying (4.2). Hence $\tilde{\mu}\left(U_{f}\right)=1$.

Theorem 4.2. For each $f \in L^{\infty}(\mu)$ its Gelfand transform $\hat{f}$ is constant a.e. $\left[\nu_{x}\right]$ for $[\mu]$ almost every $x \in X$, where $\nu_{x}$ are measures in the disintegration (4.1) of the measure $\tilde{\mu}$.

Proof. Define a measure $\omega$ as follows:

$$
\omega(W):=\int_{X} \nu_{x}(W) d \mu(x)
$$

for all Borel $W \subset Y$. If $W$ is closed-open then its characteristic function is continuous and by $(4.1)$ we have $\omega(W)=\tilde{\mu}(W)$. Then by Proposition 4.1, we get $\omega\left(U_{f}\right)=\tilde{\mu}\left(U_{f}\right)=1$ since the sets $U_{E_{n}}(n=1,2, \ldots)$ are closed-open and form an increasing sequence. Consequently $\omega\left(Y \backslash U_{f}\right)=0$ which implies the assertion in the statement of our theorem.

Acknowledgement. The authors wish to thank Professors Christian Berg, Jan Stochel and Edward Tutaj for valuable remarks.

Open Access. This article is distributed under the terms of the Creative Commons Attribution Noncommercial License which permits any noncommercial use, distribution, and reproduction in any medium, provided the original author(s) and source are credited.

\section{References}

[1] N. Bourbaki, Élements de mathématique. Livre VI, Intégration. Paris: Hermann (1959).

[2] J. Dixmier, Sur certain espaces considérés par M. H. Stone, Summa Brasil. Math. 2 (1951), 151-182.

[3] T. W. Gamelin, Uniform Algebras. Prentice Hall, Inc., Englewood Clifs, N.J. (1969).

\section{MAREK KOSIEK}

Instytut Matematyki,

Uniwersytet Jagielloński,

Łojasiewicza 6,

30-348 Kraków,

Poland

e-mail: Marek.Kosiek@im.uj.edu.pl

Krzysztof Rudol

Wydział Matematyki Stosowanej,

AGH University of Science and Technology,

Al. Mickiewicza 30,

30-059 Kraków,

Poland

e-mail: grrudol@cyfronet.pl

Received: 23 July 2011 